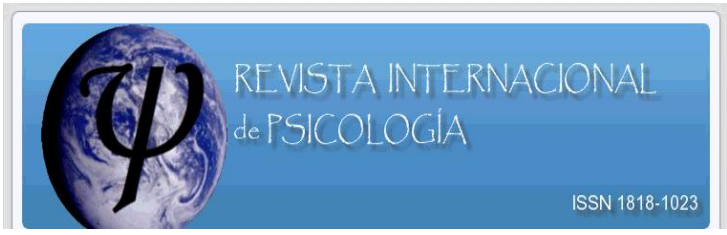

\title{
La Autopsia Psicológica en Colombia: Exploración Psicobiografica del Suicidio
}

\section{Psychological Autopsy: A Psychobiographical Exploration of Suicide}

\author{
Lic. José Alonso Andrade Salazar; Cesar Humberto Téllez; Laura Angélica Giraldo Blanco \\ Universidad de San Buenaventura Medellín extensión Ibagué
}

\section{Nota sobre los Autores}

José Alonso Andrade Salazar. Psicólogo clínico. Especialista en Gestión de proyectos de desarrollo. Docente investigador. Coordinador de investigaciones del programa de psicología de la Universidad de San Buenaventura extensión Ibagué. Investigador del Grupo Interdisciplinario para el Desarrollo del Pensamiento y la acción dialógica GIDPAD.

Cesar Humberto Téllez \& Laura Angélica Giraldo, estudiantes de último año de psicología de la Universidad de San Buenaventura Medellín extensión Ibagué. Email: chtellezc@ hotmail.com; laura-linda_15@hotmail.com

Esta investigación fue realizada con la ayuda de la Universidad de San Buenaventura Medellín extensión Ibagué.

Remita cualquier duda sobre este artículo al siguiente domicilio: Carrera $5 \mathrm{~S} \mathrm{~N} 89$ 12, Ibagué - Colombia. Oficinas de la Universidad de San Buenaventura extensión Ibagué. Correo electrónico: 911psicologia@ hotmail.com 


\section{Resumen}

Este trabajo pretende hacer una aproximación conceptual sobre los factores psicosociales vinculados al acto suicida derivados de la exploración psicobiográfica de la vida de personas que se auto eliminan, para lo cual se realizará una revisión bibliográfica del tema, con base en los aportes de artículos de revista y libros publicados en diversas fuentes de documentación. La autopsia psicología es un método de recolección de información que brinda luces acerca de las motivaciones psicosociales que originan y refuerzan el acto de quitarse la vida, actualmente es utilizado por profesionales encargados de investigar las causas del suicidio, al tiempo que los datos arrojados contribuyen en la elaboración de programas de promoción de la salud mental y prevención de ideas y comportamiento suicidas.

Palabras clave. Colombia, Suicidio, autopsia psicológica, ideación suicida, peritaje psicológico. 
The present work is a conceptual approach on psychosocial factors related to suicidal psychobiography from the exploration of the lives of people who self-eliminate, for which there will be a review of the literature, based on input from journal articles and books published in various sources of documentation. Autopsy psychology is a data collection method that provides light on the psychosocial motivations that drive and reinforce the act of suicide, and is currently used by professionals responsible for investigating the causes of suicide while contributing data produced in the developing programs to promote mental health and prevention of suicidal ideation and behavior.

Keywords: Colombia, Suicide, psychological autopsy, psychology, suicidal ideation, psychological expert. 


\section{Introducción}

Etimológicamente la palabra suicidio se remonta al latín sui y occidere, que se traduce como: matarse a sí mismo, dicha palabra surge históricamente asociada a condiciones de autoeliminación, propias de las construcciones ideológicas de cada cultura, por tanto el suicidio se ha presentado desde tiempos remotos, conservándose de diversas formas a lo largo de la historia humana (ritual-ceremonial, por honor, de purificación, como vía de escape). El fenómeno es tan complejo que ha generado múltiples visiones y perspectivas, que giran entorno a las distintas ideologías y momentos socio-históricos de la humanidad. Cabe mencionar que el suicidio antiguamente estuvo asociado a comportamientos rituales así, para algunas culturas como los romanos, el acto suicida fue una práctica honrosa y de liberación del individuo, de igual manera sucedía en Japón, con el "harakiri” o "abrirse el vientre” práctica realizada por los samurái (Herrero \& García, 2010). Asimismo, durante la Segunda Guerra Mundial, algunos pilotos tomaban la decisión de convertirse en "kamikaze", por lo que se sacrificaban al inmolarse bajo la condición ideológica de proteger su nación. Otros pueblos países que también tomaban el suicidio como un evento honroso fueron la India, los Nórdicos, los Árabes y en gran medida México con la cultura Maya, entre otros.

En la época del cristianismo San Agustín planteó que el suicidio era un "acto pecaminoso" que atentaba contra las leyes de Dios y el trascurso de la vida natural de la persona, dicho aporte también fue apoyado por gran parte de la iglesia en la edad media, 
escenario donde se impuso la idea de que aquellas personas que se suicidaran, perdían todos los derechos religiosos y cristianos. En el periodo pos medieval la legislación quitaba al suicida los derechos jurídicos, por ende todos sus bienes e inmuebles eran confiscados, al tiempo que su cuerpo era sometido a un sin número de humillaciones públicas (Sauceda \& Maldonado, 2001). Con el paso del tiempo el acto suicida se ha ido configurando como una práctica de evitación del dolor futuro, en el que se conjugan aspectos religiosos, morales, sociales y psicológicos, por ello pese a los cambios del suicidio en el transcurso de la historia, en la actualidad tiene una elevada influencia social, impactando la forma como las personas se relacionan con la muerte y con sus muertos. Un hecho interesante en la conducta suicida es el aspecto grupal, el cual incluye actos de autoeliminación colectiva, en el que individuos con mentalidad de mesías, y en función de complejos trastornos psiquiátricos, actúan como líderes ideológicos de las distintas sectas o congregaciones místicas, llegando a inducir grupalmente el acto suicida en sus adeptos (Erdely, 2000).

Para Emile Durkheim (1951) el suicidio es todo acto en el que una persona atenta contra su vida, logrando con ello su muerte de manera directa o indirecta, negativa (por efecto de disposiciones conflictivas intensas) o positiva (racionalizada en sus motivos), en cuyo fin prevalece la auto eliminación consciente. El suicidio desde esta perspectiva debe entenderse como la condición resultante de diversas fracturas en el sistema de creencias, mismas que sostienen las pautas de comportamiento tradicional (valores, principios rectores vitales, etc.), versus la emergencia e imposición política de nuevos modelos de entender la relación con el otro, y con las instituciones sociales; por tal motivo el suicidio irrumpe desde tres escenarios (de)constructivos diversos: a) como un intento de ruptura del orden social que 
excluye; b) a modo de escape ante lo inmanejable de ciertas creencias individuales y presiones derivadas de los imaginarios sociales, y c) como resultado de la constante tensión entre el estilo de vida "moderno", anclado al modelo de consumo capitalista, y la tendencia a legitimar lo tradicional como componente esencial del principio de realidad, con el que se evalúa ontológicamente las bipolaridades sociales: bueno-malo, sacro-profano, saludenfermedad, individuo-grupo, etc.; cabe mencionar que dichas variaciones permitieron a Durkheim, configurar tres tipos distintos de muerte por suicidio, las cuales presentan una relación directa con fallas en los procesos de integración con la sociedad y el individuo.

Entre los tipos de suicidio se encuentran: I) Altruista: donde se pone al grupo o ideologías por encima de cualquier cosa, y en el que las personas se rigen de manera estricta, por las normas establecidas por el entorno o sociedad, teniendo como fin la auto eliminación para ayudar a otros; II) Egoísta: en el que las personas se guían por sus propios intereses olvidando las motivaciones exógenas, al sumergirse en un ámbito más personal e individual, lo cual guarda relación con causas amorosas, enfermedades, duelos, etc.; III) Anómico: el cual se presenta cuando existe un desequilibrio de todo el ámbito social de la persona, lo que desestructura el sentimiento de importancia frente a la vida, como por ejemplo crisis económicas, guerras y violencia en aumento, exclusión y segregación social, y señalamiento histórico, entre otras (Durkheim, 1951). Uno de los elementos que llama la atención en el modelo de Durkheim, es el hecho de encontrar un patrón repetitivo en personas con vulnerabilidades sociales previas, así, sucesos derivados de fracasos afectivos, desempleo o frustraciones personales componen -anterior y actualmente- el abanico de motivaciones suicidas; dicho así para el caso de la autopsia psicológica, estos tres 
elementos, aun cuando pertenecen a una concepción explicativa tradicional, orientan al perito hacia el análisis psicosocial de las diferentes motivaciones suicidas, además de coadyuvar en el esclarecimiento, de la manera particular como cada persona otorga sentido a su acto de auto eliminación, aspectos cruciales al momento de realizar una autopsia psicológica (Shneidman, 1981).

Es importante considerar conceptualmente, que las tentativas suicidas son todos aquellos actos que no llegan a consumarse en la muerte, por lo que se relacionan con cualquier tipo de conducta intencional para atentar contra la propia vida (Pulido, Glez, Marco \& Montes, 1990). En este ámbito ingresa el concepto "parasuicidio" acuñado por Kreitman (1977) a fin de designar toda conducta auto lesiva no-mortal similar al suicidio, no reductible al hecho de "llamar la atención", pero que surge como consecuencia de problemas en el sistema de recompensas afectivas a nivel sociofamiliar, conflictos para manejar la frustración y el apego, además de una notable desmotivación para consolidar asertivamente relaciones con la otredad. Las causas del suicidio son variadas, sin embargo se resalta la influencia cada vez más progresiva y directa, de enfermedades psicológicas en función de la triada trans relacional de los siguientes componentes: emocional-afectivo-pasional, cognitivo-conductual-comportamental, y comunitario-social-institucional, además de elementos como la personalidad, dificultades para procesar la frustración, presión social, consumo de sustancias psicoactivas, eventos traumáticos, distress, antecedentes de suicidio en familiares y enfermedades somáticas especialmente. 
El suicidio en la actualidad es una de las problemáticas que a nivel mundial cobra una cantidad importante de victimas, convirtiéndose en una realidad, a la que se vinculan los distintos sectores sociales, sin tipificación de estrato social, raza o identidad sexual. Su tasa de mortalidad es tan elevada que estudios indican que en el mundo una persona se quita la vida cada 40 segundos (Mosquera, 2006), en este sentido la organización mundial de la salud (OMS, 2008), indica que en el mundo se suicidan dos personas por minuto, por ello se calcula que un millón de personas muere anualmente por dicha causa, representando una tasa de mortalidad anual de 14,5 personas por cada 100.000 habitantes, lo que a su vez solidifica la idea de que éste fenómeno, se constituye en un grave problema de salud publica, siendo una de las diez causas de muerte entre adolescentes, jóvenes y adultos (Moya, 2007). En Colombia de acuerdo con el Instituto de Medicina Legal y Ciencias Forenses (Forensis), los suicidios en el 2011 llegaron a 1864 casos, mostrando una tendencia cada vez más elevada por año; así en el 2009 fue de 1.084 y en el 2010 de 1864 personas, siendo Bogotá, Medellín y Cali las ciudades con mayor número de eventos. En el año 2011 el suicidio como manera de muerte representó el 7\% del total de muertes violentas en Colombia (Forensis, 2011), exhibiendo una tendencia al crecimiento desde el año 2006 y una relación de 4 hombres (80\%) por una mujer (20\%), y una tasa de 4,1 personas por cada 100.000 habitantes.

En éste sentido respecto a la variable edad, la entidad encontró que en Colombia la prevalencia se instala en el grupo de edad entre los 15-34 años, con un nivel educativo básico (primaria) y de estado conyugal soltero, lo cual configura un perfil del suicida análogo a las características planteadas por la OMS (2008; 2010) en otros países. Es 
importante resaltar que en gran parte de los suicidios no es posible determinar la causa y aunque en el suicida, concurran diversos elementos motivadores, los investigadores desconocen dichas causas o no la han podido determinar. Esta situación sucede a razón del escaso flujo de datos psicosociales para correlacionarlo y en general, por la falta de elementos técnicos que den cuenta de los motivos endógenos y exógenos relevantes en el suceso. Dicho esto, en la actualidad es cada vez más necesario, configurar la autopsia psicológica (AP) como un dispositivo de análisis sine qua non, adscrito a los departamentos forenses de las instituciones encargadas de investigar las muertes dudosas. En la autopsia psicológica se realiza una búsqueda psicobiográfica, que tiene como fin la recolección de datos e información, de todos aquellos eventos que posiblemente fueron desencadenantes del acto suicida como también, de patrones comportamentales de acuerdo a la personalidad, y acontecimientos que durante el trascurso de la vida del individuo tuvieron repercusiones a nivel físico, familiar, social y psicológico. Para Gómez \& Sáenz (2000) la autopsia psicológica es un método de análisis, que permite dar respuesta clara al motivo biopsicosocial de la muerte por suicidio, esclareciendo las circunstancias adjuntas, de suyo "la autopsia psicológica, es un dictamen pericial, es decir se podría constituir como prueba autónoma, por cuanto es un concepto de carácter técnico - científico" (p. 129).

Para Shneidman (1981) con la autopsia psicológica se accede a una adecuada y profunda búsqueda de los antecedentes, que orientan al profesional hacia la identificación de las intenciones del occiso, tomando en cuenta la importancia de formular las siguientes preguntas: ¿Cuál fue el detonante?, ¿Qué lo motivo? y ¿Por qué se dio en cierto momento de su vida? Estos interrogantes constituyen un punto de partida muy importante, para la 
recolección de información acerca de las distintas causas y consecuencias, asociadas al ámbito familiar y socio cultural en el que se produce el acto suicida. Por tal motivo los hallazgos provenientes de la autopsia psicológica, orientan acciones preventivas del suicidio a nivel de prevención primaria, secundaria y terciaria, las cuales buscan ayudar a la persona en riesgo, para que logre orientar su vida hacia la construcción de mejores habilidades de autoprotección, y sostenimiento emocional en el plano sociofamiliar (Morales, Buela, Bunce \& Jiménez, 2011). La autopsia psicológica en la actualidad cumple una función muy importante, y aunque tiene una elevada influencia en la parte jurídica, a menudo la falta de exigencia institucional y social del procedimiento, impide el esclarecimiento de la muerte, dificultando la construcción dialéctica de aprendizajes significativos, en el plano científico respecto al fenómeno (Morales, et al., 2011).

Para el caso de Colombia la falta de autopsias psicológicas frecuentes, influye en el hecho de que más del $50 \%$ de las variables asociadas al suicidio se encuentren indeterminadas (sin dato porcentual) en los registros de Medicina Legal (Forensis, 2009, 2010, 2011; Andrade, 2012), aspecto que permanece casi invariable en la última década. Ergo la tipificación del fenómeno es escasa, lo cual obstaculiza la construcción de modelos preventivos, ajustados a las dinámicas socioculturales y psicosociales propias de colectivos o personas en riesgo de suicidio. Cabe resaltar que en Colombia, de acuerdo a Gómez \& Sáenz (2000), la autopsia psicológica es realizada por el Instituto Nacional de Medicina Legal, así "el instrumento utilizado en Colombia para las muertes dudosas se limita a la Autopsia Médico Legal, y en algunos casos aislados se utiliza la autopsia psicológica, dejándose de lado la posibilidad de llegar a conocer por parte del juez la verdad de los 
hechos alrededor de una muerte" ( p. 8). En Colombia la AP es ordenada por un estamento penal en cabeza de un magistrado, o instituida por un organismo de salud pública en función del interés clínico-jurídico, así, en el Código de Procedimientos en Materia Penal, se aprueban procedimientos periciales a fin de esclarecer las muertes dudosas, a través de herramientas técnicas diagnósticas ajustadas a las normativas aplicables a la actividad pericial, por ello en función de la legitimidad de la evidencia física y la información "los elementos materiales probatorios y la evidencia física son auténticos cuando han sido detectados, fijados recogidos, y embalados técnicamente, y sometidos a la regla de cadena de custodia" (Título II, Artículo 277, p. 60).

Cabe mencionar que "desde el punto de vista legal, el suicidio no puede considerarse como una conducta delictuosa, aunque pueda resultar social y moralmente censurable" (Gómez \& Sáenz, 2000, p. 63). Dicho esto, debe precisarse que en el artículo 107 del Código Penal Colombiano, la inducción y ayuda al suicidio son conductas punibles por lo que toda acción que "eficazmente induzca a otro al suicidio, o le preste una ayuda efectiva para su realización, incurrirá en prisión de dos (2) a seis (6) años. Cuando la inducción o ayuda esté dirigida a poner fin a intensos sufrimientos provenientes de lesión corporal o enfermedad grave e incurable, se incurrirá en prisión de uno (1) a dos (2) años" (Código Penal, p.25). La AP se lleva a cabo por medio de la recolección documental y entrevistas abiertas o semiestructuradas a los informantes clave (Cross, Gust-Brey \& Ball, 2002), la investigación de frases, dibujos, señales o cartas de despedida, la evidencia de actos parasuicidas, frustraciones y conflictos psicosociales, perfil de personalidad, además de manifestaciones o ideación suicida previa, aspectos a los que deben sumarse, el hecho de 
entrar en contacto con el ambiente personal y social de la víctima, recrear las últimas 24 horas de su vida y realizar desplazamientos a sitios que frecuentaba (Torres, 2007). Dichos parámetros según la perspectiva psiquiátrica, deben tenerse en cuenta a la hora de aplicar el dispositivo; cabe resaltar que la autopsia psicológica en Colombia no se realiza con la rigurosidad científica con la que se hace en el resto de países puesto que, es una disciplina nueva y poco ofertada, condición que restringe la educación especializada del profesional interesado (Gómez, et al., 2000).

Las causas del suicidio arrojadas por la autopsia psicológica, permiten la identificación de eventos predisponentes, desencadenantes y mantenedores del paso de la tentativa al acto consumado. De acuerdo a Harris \& Barraclough (1997) regularmente entre las patología mentales asociadas al acto suicida se encuentran trastornos psicológicos como: I) los relacionados con el estado de ánimo como: la Depresión, Bipolar I y II, Ciclotimia, y Distimia; II) trastornos de la personalidad: Dependiente, esquizoide, paranoide, etc.; III) trastornos derivados del consumo de sustancias psicoactivas: consumo dependiente/intoxicación por consumo de Alcohol, Cocaína, Marihuana, Inhalantes, e inyectables (heroína), entre otros. En el proceso de autopsia psicológica es indispensable identificar los siguientes aspectos: edad, género, educación, calidad de la vida social y familiar, particularidad en el manejo de la frustración y los conflictos, condición económica, creencias religiosas, traumas, tipo de ideología, comportamiento y posición en los grupos de pares, e identidad sexual, ya que estas variables y categorías, son las encargadas de estructurar y modificar a través del tiempo, los distintos patrones conductuales, que tienen alguna relación con la practica autodestructiva, por lo que actúan 
como elementos resignificantes de la noción de vida en los individuos con riesgo suicida (Talcott, 2001), además de ser factores que le otorgan un volumen especial a los trastornos mentales ya mencionados (Sauceda \& Maldonado, 2001).

\section{Conceptualización, modelos y objetivos de la autopsia psicológica}

El origen del término se remonta a California a finales de los años cincuenta con Robert Litman, Edwin Schneidman \& Norman Farberow, y surge a razón de la necesidad de establecer un dispositivo de análisis para las muertes dudosas, lo cual conllevó el establecimiento de elementos metodológicos y conceptuales, que permitieran investigar por medios retrospectivos, las condiciones y características de la persona durante su vida. De igual manera, se requería plasmar los rasgos psicológicos del individuo, y así realizar una investigación sobre la importancia de eventos pasados, que pudieron reforzar o predisponer su muerte (Litman, 1989). Por otra parte Barraclough junto a otros investigadores realizaron en Europa en 1966, el primer estudio longitudinal de autopsia psicológica, a razón de 100 eventos de suicidio en Inglaterra. Es importante resaltar que Cuba, es uno de los países con mayor profundización en éste método de investigación psicológico, y gracias a su Ministerio de Salud Pública, desarrolló un formato para su realización, el cual se denominó "Modelo de Autopsia Psicológica Integrado" (MAPI) creado y validado por la doctora Teresita García Pérez (1993; 1994), en el que se reúnen datos de la víctima, familiares y personas cercanas, a fin de recolectar elementos probatorios sólidos, y rasgos 
característicos del suicida que permitan esclarecer sus motivaciones (Vidal, Pérez \& Borges, 2006).

Éste tipo de métodos son regularmente utilizados por peritos expertos, encargados de explicar el contexto psicológico y social del fallecido, así puede ser usado para entender las conductas criminales y auto destructivas de ciertos individuos. Dicho esto, es indispensable resaltar que éste profesional direcciona todos los hallazgos de la AP, hacia explicaciones psicológicas y sociales, que sirven de base para aclarar aspectos afectivos y jurídico-legales de la muerte, además de identificar puntos críticos en las motivaciones y posibles causas adyacentes. Per se, durante el proceso investigativo la estrategia de análisis propia de la autopsia psicológica, requiere de trabajo interdisciplinario con criminalistas, técnicos judiciales, abogados, fiscales, médicos forenses y legales, trabajadores sociales, psiquiatras y psicólogos jurídicos, siendo el equipo transdisciplinar el encargado de recolectar información veraz acerca de los datos del individuo que se auto elimina, lo que permite la creación y estructuración del perfil psicológico y mental del occiso (Acevedo, Núñez \& Pinzón, 1999). En algunos países como España, Inglaterra, Estados Unidos y Cuba, la autopsia psicológica es sumamente importante, pues es tomada como una herramienta investigativa a nivel biopsicosocial de los individuos que fallecen, lo que permite dar claridad a tres tipos de muerte: suicidio, homicidio y accidentes de tránsito, que se presentan en un espacio y tiempo específico (Bárcenas \& Mardones, 2006). En Colombia la AP es un procedimiento que como método de investigación psicoforense, se encuentra limitado en su praxis por la falta de información y conocimiento profesional (Gómez \& 
Sáenz, 2000) lo que dificulta acceder a una validez y confiabilidad de las causas biopsicosociales asociadas al suicidio y parasuicidio.

Cabe mencionar que por medio del Acto Legislativo 03 de 2002, se incluyeron reformas a los artículos 116, 250 y 251 de la Constitución Política de Colombia (CPC), con la finalidad de "establecer un nuevo sistema de investigación, acusación y juzgamiento en materia penal" (USAID \& Defensoría del pueblo, 2006, p. 14), por tal motivo en el Código de Procedimientos Penales (CPP, Ley 906 del 2004) se mantuvo la distinción entre la "fase de investigación - encaminada a determinar si hay méritos para acusar- y la fase de juzgamiento" (Sentencia C - 873 de 2003), escenario en el que se dio una importancia especial a aspectos de la defensa como: la oralidad (incluye testimonios de "peritos" expertos en salud mental y resultados de autopsia psicológica) (Artículo 268), publicidad, inmediación de pruebas (Artículo 333 de la Ley 906 de 2004) en las que se puede incluir información derivada de la evaluación forense (CPP, Libro II Título I Capítulo I), la contradicción, la concentración y el respeto por todas las garantías fundamentales de los participantes en la investigación (Sentencia C - 873 de 2003), para lo cual se requiere de un cuerpo técnico de Investigación Defensorial (Ley 941 de 2005, artículo 36), el cual debe hacer uso de todas las herramientas periciales, para demostrar las causas y consecuencias asociadas al acto de muerte, por lo que "los laboratorios de criminalística del ente acusador serán examinados y controvertidos en el momento del juicio; muchos serán testigos y como tales, obviamente, estarán frente al Juez, donde se les interrogará sobre su actuación desestimando la prueba de cargo" (USAID, et al., 2006, p. 14) 


\section{Modelos de autopsia psicológica}

De acuerdo a Shneidman (1981) no existe un modelo establecido o fijo que de cuenta de las causas absolutas de la muerte de un sujeto, ya que dicho componente se estructura a partir de las vivencias socio-históricas de cada lugar, en consecuencia los investigadores ajustan sus estrategias de análisis a los diversos contextos psicosociales donde se produce el hecho, determinando cual será el procedimiento a utilizar para la recolección de los datos acerca de la muerte del sujeto. Estas afirmaciones dadas por Shneidman generaron oposiciones con algunos investigadores, ya que para ellos el modelo no era confiable debido a su falta de validez y estructura, lo cual lo llevaba a tener un sesgo bastante marcado. Por esta razón, Annon (1995) intenta crear un modelo de AP que tenga mayor objetividad y que por ende conserve una estructura, en la que se presenten lo siguientes pasos: I) Hacer una revisión minuciosa de los distintos acontecimientos que se dan en la escena; II) Interpretar de manera detallada los elementos recolectados por medio magnético, como es el caso de fotografías y filmaciones, las pruebas documentadas, testimonios de los distintos implicados (testigos, familia, conocidos, entre otros), y reportes técnicos de las entidades que tienen relación directa con el estudio del caso; III) Búsqueda y recolección de elementos como historia clínica, antecedentes biográficos escritos, cartas y otras evidencias sentimentales, que arrojen información del porqué sucedió el evento; IV) Tener en cuenta que el tiempo empleado, antes de dar un dictamen hipotético debe ser entre 20 y 30 horas, lo cual hace parte de las habilidades del investigador para aproximarse a las causas de muerte (Annon, 1995). 
Los dos modelos planteados sirvieron como base para la formación del Modelo de Autopsia Psicológica Integrado (MAPI) que según la Dra. Teresita García Pérez (1998) autora del mismo, es un elemento que posee una estructura cerrada, y evita el sesgo en los análisis postmorten. El MAPI fue creado en Cuba entre 1990 y 1996, con el fin de analizar los tres tipos de muerte que se presentan con mayor frecuencia: el suicidio, el homicidio y los accidentes de tránsito. A nivel forense, en el análisis de las victimas de suicidio se transita por tres etapas: la primera de ellas implica crear un escenario retrospectivo que posibilite recolectar y depurar la información del deceso. Dicha búsqueda se hace de manera individual y en privado, luego se procede con la revisión del expediente medicolegal, para entender aspectos etiológicos, la postura de la muerte y la implicancia de personas que pudiesen tener conocimiento del hecho. Lo segundo conlleva la aplicación de instrumentos forenses diseñados para el estudio de personas que se hayan auto eliminado, y la tercera es la generación de métodos concluyentes que reúnan la información de los dos puntos anteriores. Asimismo, resulta necesario tener en cuenta que es óptimo recolectar la información lo más rápido posible (Núñez de Arco \& Huici, 2005), tiempo que puede variar entre uno y tres meses (Caballero, et al, 2010), entre seis meses y un año máximo (García Pérez, 1999; 1999a), e incluso hasta varios años (Cross, Gust-Brey \& Ball, 2002) después de la muerte del individuo. Cabe anotar que a pesar de que las posiciones respecto al tiempo de recolección de la información pueden ser divergentes, es recomendable que dicho tiempo sea análogo al periodo de duración del duelo, ya que entre más pronta sea la adquisición de la información, menos son los sesgos explicativos y las resistencias 
psicológicas en los entrevistados, lo cual ayuda a esclarecer los antecedentes sociales, y aspectos emocionales relacionados con el suicidio (García Pérez, 1993).

En el caso de homicidio según la doctora Teresita García Pérez (1995), el proceso de autopsia psicológica se ejecuta por medio de estudios socio-patológicos que sé dividen en cuatro etapas: a) Crear o implementar un instrumento, que permita realizar una búsqueda de información de las personas que hayan sido victimas de homicidios; b) Demostrar la validez y confiablidad del instrumento, gracias a la información recolectada; c) Identificar por medio de entrevistas o trabajo de campo, la relación que tenia la victima con los posibles autores del suceso; y d) La reconstrucción de los posibles hechos por medio de restructuración de la escena. Por otra parte Jiménez (2001) plantea que en los accidentes de transito se utiliza el MAPI, pero con una mayor recolección de información, que tenga relación directa con el tipo y la forma del accidente. Análogamente el MAPI tiene en cuenta a nivel general las siguientes etapas: a) Recolectar huellas psicológicas dejadas en la escena donde aconteció el evento; b) Realizar el proceso de entrevista como mínimo a tres de los parientes y conocidos; c) Discutir a nivel de foro para interpretar el hecho con los distintos profesionales que tienen relación directa e indirecta con el proceso (Burgos, 2006). El MAPI es el método de investigación psicosocial de muertes dudosas, más utilizado en el campo forense, a razón de que su elevado índice de validez y confiablidad, permite disminuir el error en la información, evitando el sesgo en los datos recolectados, lo que marca una evolución de modelos tradicionales, cuya estructura evaluativa era débil y variable. 
Para el caso de Colombia la AP es un procedimiento de uso privativo del Instituto de Medicina Legal y Ciencias Forenses, que se adjunta a la praxis de los peritos forenses especializados en protocolos para el manejo e investigación en casos de homicidio y suicidio, o en todo tipo de muertes dudosas, lo cual limita las posibilidades de acceso al dispositivo desde planos no legislativos. Un hecho interesante es que el escaso uso del procedimiento en todos los casos de suicidio, puede estar asociado al escaso interés de los profesionales y el poco conocimiento de la familia acerca de su aplicabilidad (Gómez et al, 2000); sin embargo los datos arrojados de casos evaluados en Colombia indican la prevalencia de rasgos de personalidad con tendencia a lo depresivo y paranoide (Nuñez, 1999) como también, problemas afectivos de base (relación de pareja, autoconcepto, problemas sociales diversos) e información acerca de la preferencia por ciertos mecanismos de muerte tales como, el ahorcamiento (mayor en hombres), proyectil de arma de fuego e intoxicación (mayor en mujeres) en ambos géneros (Forensis 2011) . Es importante resaltar que una aproximación a un modelo de autopsia psicológica para Colombia, debe incluir aspectos socioculturales propios de las comunidades en las que se presentan dichos eventos (religión, ideologías, códigos lingüísticos y creencias acerca de la muerte) puesto que, el análisis del suicidio implica entender "las circunstancias concretas (geográficas, económicas, culturales, etc.) presentes en cada una de las sociedades estudiadas por la antropología jurídica” (Juanatey, 1994, pp. 32-33), además del estudio de la permanencia de elementos generadores de un elevado estrés como por ejemplo, desplazamientos forzados, acoso rural y urbano de grupos armados, brutalización, pandillismo, violencia intrafamiliar y urbana, Bullying, negligencia, maltrato y abuso sexual, catástrofes previas de tipo natural o provocada, y actos coercitivos de carácter generacional anclados al sistema 
de creencias, y a los imaginarios sociales de las víctimas y su comunidad de base, aspectos han sido tomados en cuenta en las estandarizaciones del MAPI en países como España y Argentina, sin una correlación o interés específico en cuanto adaptación del instrumento para Colombia (Bárcenas \& Mardones, 2006).

\section{Objetivos de la autopsia psicológica}

La autopsia psicológica tiene como objetivo principal, esclarecer y definir la motivaciones individuales, y las causas biopsicosociales asociadas a la muerte por suicidio, homicidio y accidente de tránsito, sirviendo de material probatorio en el aspecto jurídico, y como elemento de sostén sociofamiliar en casos de duelo o inconformidad, con los supuestos que configuran el acto de muerte (Scott, Swartz \& Warburton, 2006). Por ello en los procesos de estudio de caso, la autopsia psicológica, toma en cuenta las conductas externas de los individuos con ideación suicida, ya que al préstales atención sirven como fundamento para descubrir los indicadores de riesgo suicida, mismos que emergen desde lo latente hacia lo manifiesto, a través de desencadenantes sociales específicos para la vida del sujeto. Entre estas condiciones se encuentran manifestaciones analógicas -no expresadas de manera verbal-, las cuales evidencian variaciones conductuales a nivel alimenticio, que desencadenan diversos trastornos digestivos (Strock, 2001). Lo anterior fortalece la tendencia a la evitación de situaciones publicas, en las que se requiere tener contacto con pares, pareja, familia o con desconocidos. Esta situación suele ser tan dañina, que motiva en muchas personas el abandono personal, la huida del encuentro con la otredad, problemas de 
auto concepto y el no-cumplimiento de actividades estudiantiles y laborales, constituyendo paulatinamente un patrón de humor depresivo, con tendencia a la evasión y el retraimiento.

De suyo, la AP regularmente encuentra que las personas que cometen suicidio, tienen un incremento del consumo de sustancias psicoactivas, cuadros somatomorfos de tipo crónico, historial de exposición a circunstancias en las que se arriesga la vida además, de alteraciones fisiológicas importantes como, problemas musculares, cefalea tensional, dificultad para encontrar los niveles adecuados de sueño, depresión, alucinaciones auditivas, y preocupación constante por la muerte (Acevedo, et al., 1999). El siguiente elemento incluye manifestaciones que requieren expresión verbal o escrita, por parte del individuo que va a consumar el hecho, por ello la persona previamente ha manifestado sus deseos, llegando a pasar desapercibido por sus familiares quienes lo asocian a un estado de manipulación o chantaje emocional. Entre las frases más usadas están: "quiero acabar con mi vida", "tengo ganas de suicidarme", "muy pronto dejare de ser una dificultad para ustedes", "presiento que me voy a morir", "si algún día no vuelvo a la casa solo recuérdenme", "es mejor estar muerto que vivo", "siempre los recordare", "la muerte es lo mejor que me puede pasar", "quiero que le den esto a ...", "esto que me gustaba ya no le encuentro importancia, alguien lo necesita o lo arrojo a la basura", "quiero un cuaderno para escribir mis memorias", estos elementos evidencian un elevado descontento con la vida, en el que la persona no se siente a gusto con ningún evento que acontece en su entorno (Sauceda \& Maldonado, 2001).

De acuerdo a lo expuesto, la AP genera cuatro objetivos primordiales (Ebert, 1991): I) 
Esclarecer si la muerte es de tipo natural, accidental, suicidio u homicidio, lo cual se puede identificar a través del acrónimo "NASH"; II) Tipificar de la edad del occiso, la sucesión cronológica de eventos predisponentes, y el ambiente en el que efectuó sus interacciones, en este momento de la AP, se tiende a recolectar la mayor información posible de los distintos hechos que acontecieron en la vida del sujeto, y que tienen alguna relación directa o indirecta con la causa del deceso; III) Velar por obtener información verídica, para identificar la diversidad de los hechos suicidas, y así gestar propuestas y campañas preventivas para la disminución de su letalidad; IV) Convertirse en un elemento terapéutico apto para la confrontación del duelo de los familiares, allegados y conocidos. Por tanto los procesos investigativos y de entrevista de la autopsia psicológica, se utilizan también, como factores terapéuticos ya que, sus funciones permiten confrontar con la familia, algunos eventos emocionales y psicológicos suscitados por la muerte de un allegado. La AP tiene un efecto terapéutico en las familias de suicidas, logrando disminuir los sentimientos de negación, culpa, ira, tristeza y dolor que las personas albergan, al entregar a cada nucleo familiar respuestas acerca de las motivaciones biopsicosociales que impulsaron el acto suicida.

La autopsia psicológica tiene la función de esclarecer las muertes dudosas, llegando a explicaciones veladas para el ojo no experto, para lo cual se vale de las habilidades de diversos profesionales entre los que se encuentran, técnicos expertos en criminalística, peritos, médicos forenses, y profesionales encargados de la parte legal (Delgado y col., 1994). De modo descriptivo se enumeran a continuación, siete condiciones de la praxis requeridas al momento de realizar una AP: I) Valoración de los distintos riesgos, tanto 
homogéneos como heterogéneos, que pueden llegar a ser parte directa de los eventos (Talcott, 2001); II) Entender el estado mental del individuo en el momento de la muerte; III) Tipificar el nivel y estilo de vida de la persona antes del suicidio; IV) Reconocer la afectación de las áreas a nivel emocional, motivacional y por ende, los conflictos ocultos o aparentes, con un amplio significado en la vida del individuo; V) Estructurar el perfil de la victima a través de la información recolectada; VI) Reconocer sí durante su vida, la familia percibió señales que pudieron apuntar al evento suicida; VII) Establecer si se apreciaron previamente estado presuicidas o tendencias parasuicidas. Estos elementos se convierten en una guía que orienta el análisis a seguir, a fin de entender la letalidad del acto suicida y sus implicaciones previas y actuales en la familia, la comunidad y la sociedad, ya que los patrones conductuales y comportamentales arrojados por dichos estudios, sirven de base para la comprensión de las tendencias epidemiológicas acerca del suicidio.

De manera complementaria Young (1992), establece una serie de pasos importantes para realizar un proceso de autopsia psicológica adecuado, entre los que se encuentra: el estudio de la identificación de todos los datos personales e íntimos del individuo, la recolección de detalles que permitan identificar los motivos de la muerte del sujeto, como también de familiares con muertes dudosas; otros aspectos son el contar con respuestas claras de sus parientes en relación a eventos generadores de estrés, lo que implica entender la dinámica familiar, la identificación de miembros que presenten consumo de sustancias psicoactivas, al tiempo que el modo como se confrontan y manejan las distintas relaciones interpersonales (Heikkinen, Loenqvist, 1995). Otros aspectos son los eventos fantasiosos, la identidad e inclinaciones sexuales, la frecuencia de algunas conductas autodestructivas 
durante la vida, y finalmente el tipo de respuesta de la familia y otros parientes ante las manifestaciones digitales o analógicas de auto eliminarse. La autopsia psicológica busca comprender per se, la intencionalidad del acto suicida, lo cual posibilita una aproximación al grado de letalidad implicado en la conducta suicida (Peck \& Warner, 1995). Igualmente Annon (1995) aporta aspectos complementarios como por ejemplo, el realizar una revisión minuciosa y profunda, con un alto nivel de observación de la escena donde acontecieron los hechos, apoyándose de elementos magnéticos (fotografías, videos, etc.) además de referencias de seguridad, salubridad y confiabilidad del lugar de muerte, conjuntamente a eventos asociados a situaciones de riesgo toxicológico (Appleby y col., 1999; Cheng y col., 1997).

Es preciso resaltar que la Autopsia Psicológica cuenta con dos utilidades prácticas, por ello como instrumento de investigación permite esclarecer el tipo de muerte dudosa, gracias a la información recolectada sobre los datos psicológicos del occiso, de igual manera se utiliza para estudios de victimología y vulnerabilidad psicosocial; la segunda utilidad indica que como instrumento psicológico, ayuda a la predicción y prevención de eventos de riesgo suicida, que pueden emerger en el transcurso de la elaboración del duelo de los familiares. Según Isometsa y col., (1996) la autopsia psicológica debe contener preguntas dirigidas al esclarecimiento de los hechos, y para ello debe contar con una estructura epistemológica adecuada además, de habilidades dialógicas en los entrevistadores, capacidad empática y vocación investigativa. 


\section{El rol del psicólogo en la autopsia psicológica}

El psicólogo que forma parte del equipo encargado de aplicar la autopsia psicológica, debe desarrollar destrezas que le permitan entender el suicidio, como una problemática social cuyo impacto altera la calidad y expectativa de vida de personas y comunidades, lo cual lo constituye en un problema de salud publica de innegables magnitudes epidemiológicas. Esto obliga a que el experto cuente con conocimientos psicológicos y jurídicos, dirigidos hacia el ámbito legal y la orientación familiar, para lo cual requiere contar con una adecuada capacidad de escucha, habilidades de comunicación individual y grupal, además de un alto nivel de afinidad hacia los procesos investigativos (Aja, 2009), asimismo tener curiosidad y duda ante lo "evidente", por lo que el psicólogo se entrena en la lectura de las distintas señales, que pueda proporcionar la escena del crimen. Para Shneidman (1994), contar con amplia agilidad en los procesos de entrevistas, permite que la información pueda fluir de una manera fácil y profunda es decir, consintiendo la catarsis del informante, orientándolo hacia el esclarecimiento del suceso, al preguntar de forma directa sin caer en exacerbaciones del tema, o forzando las resistencias psicológicas del entrevistado. En términos generales el profesional tiene la obligación de asesorar, evaluar y especificar el acto suicida a partir del conocimiento conductual, personal y emocional del occiso, generando hipótesis acerca de las motivaciones del suceso.

El psicólogo debe tener buenas relaciones interdisciplinarias con profesionales como abogados, jueces, criminalistas y peritos, también con personal experto en el área de la 
salud como, médicos generales, forenses y legales, psicólogos jurídicos, forenses y psiquiatras, con los cuales puede realizar una eficiente asesoría profesional (Delgado y col., 1994). En este aspecto tener apropiadas relaciones interpersonales, incluye la habilidad para comunicarse asertivamente con familiares de la victima, y otros relacionados social y afectivamente con el occiso (Acevedo, Núñez y Pinzón, 1999). Según Acosta (2011), el rol de la persona que ejecuta la AP, implica el uso del psicodiagnóstico, puesto que a través de éste, es posible realizar una exploración indirecta de la personalidad del suicida. Dicha técnica se desarrolla por medio de una evaluación psicológica, con la que se busca identificar el perfil de la victima en cuanto comportamiento, estado mental y dinámica vital (vida social, psicológica y biológica). En consecuencia el rol del psicólogo en la autopsia psicológica se constituye per se, en función de la practica profesional de la psicología forense, en el marco de las habilidades ya nombradas, y a partir de condiciones psicosociales propias del entrevistador y del entrevistado, lo que constituye un campo de acción en el que se delimitan los aspectos éticos, prácticos y profilácticos de la intervención.

Para Shneidman (1981), la muerte debe ser analizada por el profesional encargado de hacer la autopsia psicológica de acuerdo a tres aspectos: "el qué", o espacio en el que se indaga el suceso y los eventos desencadenantes de la situación previa a la auto eliminación; "el cómo", en el cual se identifica el desarrollo y consumación del hecho, para ello se toma en cuenta la ideación suicida previa, y por ende su proceso hasta el suicidio consumado; y "el por qué", aspecto que busca entender las razones que llevaron a la persona a cometer el suicidio. Cabe resaltar que en este punto, el profesional debe tener la habilidad de analizar 
la esfera emocional y mental del individuo. Estos tres aspectos le permiten al profesional la generación de hipótesis aproximativas al suicidio, que actúan como elementos coadyuvantes del esclarecimiento del motivo, y las circunstancias biopsicosociales asociadas al acto suicida. El rol del psicólogo presenta objetivos concretos en cuanto a intervención, que se dirigen a la búsqueda de la confiabilidad de la información recopilada, además del acercamiento y contacto con las victimas, por esta razón resulta crucial realizar una depuración de las diversas fuentes de información, con el objetivo de cruzar los datos obtenidos y ofrecer seguridad en las hipótesis. Lo anterior se decanta respectivamente, en la construcción de acciones interdisciplinarias preventivas, que reducen la elevada incidencia del fenómeno en la sociedad (Shneidman, 1967).

De acuerdo con Ebert (1991), el profesional debe realizar el proceso de la AP de manera ética y competente, indagando aspectos complejos de las relaciones intrafamiliares, al igual que condiciones problémicas derivadas de las relaciones a nivel social y comunitario; en estos tópicos la ética y el buen manejo de la información, son condiciones sine qua non del trabajo investigativo, tomando en cuenta que la posibilidad de acceder al historial psicopatológico adscrito a la historia clínica (Conwell y col., 1991; Asgard, 1990), es uno de los puntos base del diagnostico en la autopsia psicológica. El psicólogo en estos escenarios además de servir de mediador, entre la institución judicial y la familia del suicida, tiene la obligación profesional de contener los desbordamientos emocionales, motivados por la catarsis emergente de la reviviscencia de los sucesos traumáticos. El rol del psicólogo en este tipo de procedimientos, conlleva la estructuración de una personalidad empática con el dolor del otro, con un profundo sentido social y una capacidad de análisis 
importante, lo que indica una puesta en marcha de habilidades sociales, en las que prima la curiosidad, el asombro, la objetividad y el factor ético, como fundamentales para entender la dinámica de los actos suicidas. Todos estos elementos permiten un acercamiento significativo a la compresión del fenómeno del suicidio, lo cual es coadyuvante en la elucidación de los eventos de muerte, por lo que sus resultados se convierten en insumos, que fortalecen la praxis profesional y el desarrollo de la autopsia psicológica, como una técnica cada vez más avanzada, confiable y verídica (Ebert, 1991).

\section{Discusión}

Habitualmente la autopsia psicológica es utilizada como herramienta evaluativa, a razón de la bondad, versatilidad y confianza de los datos que sus categorías de análisis arrojan, lo cual no reduce su función a una mera categorización de signos, síntomas o alteraciones en las relaciones sociales de la victima; por tal motivo más que un instrumento descriptivo, la AP actúa como dispositivo de prevención, especialmente porque desde una postura longitudinal en el análisis de los datos, es posible tipificar las tendencias epidemiológicas de los factores de riesgo asociados al acto suicida, lo cual fundamenta a modo de línea base, la creación de proyectos y programas de promoción de la salud mental positiva, y prevención de las conductas de riesgo para la vida, a fin de fortalecer la calidad y expectativa vital de las comunidades y personas en riesgo de suicidio. Según Barradough y col., (1974) mas de la mitad de personas que se suicidan dan a conocer ésta idea a la familia 
de diversas formas, que van desde la manifestación verbal, hasta demostraciones simbólicas a menudo imperceptibles por el núcleo familiar y social, así mismo otras personas evidencian sus planes sólo hasta el momento previo al suicidio, lo cual limita la posibilidad de regulación y control de la acción autolítica. En éste argumento el punto central es que el suicidio es una conducta que se puede prevenir, cuando se cuenta con la información de riesgo proveniente de eventos previos, y el reforzamiento constante de factores protectores emergentes de la interrelación cooperativa entre familia, comunidad e instituciones.

De suyo, el uso de la AP en estudios epidemiológicos permite el conocimiento del suicidio desde un escenario multifactorial, en el que la corresponsabilidad no se limita al plano sociofamiliar, pues sus causas se estructuran en gran medida a partir de las desigualdades sociales, propias de la relación histórica entre la comunidad y un estadonación, en el que prima el desempleo, el incumplimiento estatal, la impunidad y la legitimidad de la muerte, por lo que el suicidio estaría configurado como una de las formas especificas de ruptura del ordenamiento social excluyente y autocrático. En gran medida los factores de riesgo no son solamente el resultado de eventos desencadenantes del suicidio, sino la conjunción dinámica de condiciones de peligro y alienación vital a nivel histórico, en las que prima la muerte como estrategia de escape ante la presión política-social, misma que caracteriza la dinámica coercitiva de los estados sociales actuales (Agamben, 1998). Dicho esto, la autopsia psicológica es también un dispositivo de análisis sociocultural, ya que la condición psicológica y social del sujeto que se suicida, es en gran medida el correlato del funcionamiento inapropiado, de la interacción (interrelación, interdependencia, desacople y reorganización social) entre personas, comunidades e 
instituciones, relación que emerge en la dinámica interaccional, entre los espacios naturales y artificiales donde dichas vinculaciones germinan (Bateson, 1999).

Según Jiménez y col., (2002) la AP es un método que sirve como fuente epidemiológica de información confiable, acerca del acto suicida y su relación con otras condiciones que cultural y socialmente, pueden ser consideradas como un peligro para la salud mental y física, de quienes se encuentran en riesgo posible o evidente de suicidio, así esta condición enfrenta su principal reto, al tener que ajustarse a los parámetros interpretativos con los que la familia y la comunidad, da sentido a la autoeliminación del otro; de suyo dependiendo del contexto en el que dicha lectura del fenómeno emerja, se producirá también una reacción interpretativa, por lo que a pesar de que el instrumento de análisis, que en este caso puede ser el MAPI, brinde todas las garantías de objetividad para evitar el sesgo al momento de interpretar las circunstancias de muerte, la esfera sociocultural define las condiciones simbólicas que cada familia y la comunidad otorga al deceso, lo cual le da un giro exegético al dispositivo. En éste sentido la autopsia psicológica como dispositivo analítico, consiente la tipificación de factores de riesgo evidentes en las dinámicas intra e intercomunitarias, de las poblaciones bajo diversas condiciones de vulnerabilidad (Talcott, 2001). Cabe mencionar que aunque estos argumentos resalten la importancia de la AP en la prevención del suicidio, la ideación suicida y el parasuicidio, en países como Colombia dichas intervenciones no representan una condición sine qua non de la investigación forense, llegando a limitarse a una búsqueda de información superficial que adolece de profundidad, en cuanto a los factores socio familiares y psicosociales asociados al acto suicida. 
En Colombia la falta de garantías económicas para los profesionales de la salud que ejercen en escenarios judiciales, sumado a la deficiente oferta de especializaciones acerca del tema, y el desinterés de quienes no encuentran en la autopsia psicológica una fuente de ingresos constante, ( Torres \& Luna , 2008), limita la implementación operativa de estas practicas, situación análoga a la deficiente demanda del servicio especializado por las autoridades judiciales, y la falencia del gobierno al no-legitimar éste servicio, como indispensable para esclarecer no solo los delitos, sino también las muertes que por su condición de duda, congestionan el sector judicial, y deterioran las garantías legales de los implicados. De igual manera, la falta de profesionales que cubran competentemente la demanda asistencial, hace que el servicio se privatice, generando un sistema de oferta y demanda, donde aquellas familias que cuentan con la capacidad adquisitiva para cubrir los gastos de dichos estudios, acceden al esclarecimiento necesario para procesar el caso, ejercer presiones sobre los implicados o ajustar sus interrogantes biopsicosociales, a las condiciones explicativas que la AP genera. Nordstroem y col., (1995), aseguran que cuando en el historial de una persona existen antecedentes previos de ideación suicida, parasuicidio y/o intento de suicidio, sumados a trastornos psicopatológicos, el riesgo de suicidio es elevado, constituyéndose en predictores confiables de dichas conductas. Según Pérez (1999), es importante mencionar que no todas las personas que tienen trastornos mentales se suicidan (Pérez, 1999, Tuesca \& Navarro, 2003), y que la correlación con antecedentes previos de intentos de suicidio no es una condición sine qua non pues, aquello que realmente suscita la autoeliminación, es la progresión de dichos componentes a estructuras 
comportamentales cada vez más complejas, en las que la vida se deslegitima abriendo paso a la perdida del sentido vital.

De acuerdo a lo expuesto, a pesar de que el aspecto psicopatológico puede ser asumido como primordial al momento de encontrar una causa científica al suicidio, el fenómeno no se reduce a la descripción o categorización patognomónica, por lo que es necesario correlacionar variables como edad, género, duración de la enfermedad y presencia de desórdenes afectivos (Ahrens y col., 1995). Per se, ambas posiciones son reduccionistas, y la AP es muestra de la necesidad de desligarse de posturas unilaterales y objetivistas, que someten el suicidio a las emergencias históricas heterocíclicas, generalizando estas conductas en función de la repetición social, de ciertos componentes de riesgo con un valor transgeneracional importante. Por tal motivo lo que la autopsia psicológica propone, es la posibilidad de entender los contextos sociales y sus dinámicas intrínsecas, a partir de elementos que sólo la familia y el circulo social del suicida pueden compartir con el evaluador, siendo en ultimas cuentas la familia, la comunidad y la sociedad a través del dispositivo de análisis, quienes otorgan sentido a las causas y consecuencias asociadas al deceso. Dicho así, la AP permite diseñar y operar, distintas estrategias preventivas en las familias y comunidades en las que se aplica, gracias a la identificación del perfil psicosocial y las habilidades sociales y metacognitivas alteradas en el suicida. La AP como dispositivo social posibilita la identificación de población en riesgo suicida, lo cual tiene un efecto de reducción de la tasa elevada de mortalidad por suicidio a edades cada vez más tempranas (García Pérez, 1998). 
En Colombia se pude identificar que pese a la continuidad e incidencia del suicidio, las autoridades forenses aplican el método de AP de manera poco rigurosa y científica, debido a la falta de información sobre el método, y la escasa demanda del servicio (Gomez \& Saenz, 2000), aun cuando es coadyuvante en el esclarecimiento de casos dudosos de muerte, además de colaborar en la identificación de rasgos psicológicos y perfiles suicidas, necesarios para la evaluación, diagnóstico, tratamiento y seguimiento de individuos que atentan contra su propia vida. Es relevante afirmar que aunque el procedimiento de AP no siempre determina con exactitud si la muerte fue suicidio, homicidio o accidente, sus hallazgos facilitan la tipificación de la muerte al tiempo que, determina sus implicaciones previas, actuales y futuras (Eur Psychiatry, 2002). Un elemento crítico es la subjetividad impresa por el analista al momento de realizar la entrevista, encuesta o la observación participante, lo cual se supera cuando el dispositivo analítico presenta una objetividad y consistencia interna, permitiendo consignar todos aquellos aspectos que no por ser culturales, o históricamente determinados por la tradición, dejan de ser importantes para el análisis del suicidio. Si bien la AP podría ser más efectiva y eficaz en el plano grupal (optimizar recursos y procesos), el carácter de encuentro de individualidad le otorga al proceso investigativo, la validez suficiente para acercarse a la etiología social del suicidio, lo cual es dable bajo normas éticas y estéticas, en las que se vela por la dignidad y el beneficio del ser humano, evitando la coerción de los distintos derechos y deberes del occiso y de sus familiares.

Cabe mencionar que estas acciones en muchos casos, no son tomadas en cuenta por el entorno social y familiar del individuo, debido a mitos varios como por ejemplo, la idea de 
que alguien que se va suicidar no lo anuncia. Sin embargo la AP evidencia que estas personas mantuvieron a sus familiares al tanto de sus ideas, a través de quejas, actos simbólicos y cambios comportamentales, que en un escenario de acostumbramiento conductual pueden pasar desapercibidas; ergo la educación preventiva y el refuerzo social de la protección comunitaria en familias y comunidades, puede ayudar a detectar de forma temprana situaciones de riesgo suicida (Aja, 2009). Es importante resaltar que para que los procesos de prevención en Colombia sean más eficaces y efectivos, es necesario trabajar con los núcleos familiares, ya que estos escenarios logran brindar el soporte afectivo y la estabilidad psicológica necesaria, para controlar y resignificar positivamente la conducta suicida, lo que puede disminuir las tasas de suicidio. Por esta razón, es importante que se utilice con mayor frecuencia y rigurosidad el modelo de la AP en los casos de suicidio, ya que con ella se tendrían mayores herramientas para la realización de proyectos preventivos. La AP ejemplifica lo limitante de los sistemas interinstitucionales de protección a la vida, además de las desigualdades históricas en los grupos, en su mayoría vulnerables a nivel socioeconómico; del mismo modo como técnica puede aproximarse al sentido de las conductas premorten y el perfil psicosocial del suicida, pero no puede determinar su sistema de contingencias personales, por lo que la ejemplificación de las causas de muerte, no es el único criterio para validar el constructo explicativo de la AP, lo cual invita a la reflexión antes que a la categorización de la conducta de muerte. 


\section{A modo de corolario}

La relevancia del estudio de la vida de la persona suicida a través de la AP, se da con base en la necesidad de disminuir la tasa de mortalidad por esta causa, por ello resulta imprescindible revisar la condición psicológica de la victima, la estructura de personalidad, la fuerza del yo, el tipo de apoyo familiar y social, además del nivel de desesperanza previo y las experiencias de dolor y perdida que anteceden a dicha decisión; estos aspectos pueden buscarse en el núcleo familiar y social, a fin de localizar correlaciones que apunten a la satisfacción/insatisfacción de necesidades biopsicosociales en sus territorios de socialización significante. Por otra parte, en cuanto al perfil, objetivos y pruebas psicológicas frente a esta problemática, es importante resaltar que dicha trilogía aun se encuentra en investigación, debido a que éste es un tema nuevo en cuanto métodos y técnicas a implementar a la hora de llevar el caso desde un escenario transdisciplinar. Dicho esto el MAPI es un modelo de autopsia psicológica que como método, técnica y dispositivo social, pose una estructura de análisis que evita el sesgo respecto a las causas, sirviendo como módulo de análisis de la multiplicidad de factores asociados al acto suicida, el homicidio y los accidentes de transito.

El suicidio es una de las problemáticas sociales cuya necesidad de prevención es innegable, lo cual constituye una de las tareas más arduas que el estado y las instituciones sociales tienen. En Colombia pese a que se encuentran muchas investigaciones acerca de éste tema, se han hecho pocas campañas o estrategias de promoción y prevención desde la 
$\mathrm{AP}$, pues las tácticas de divulgación de la información preventiva, pueden resultar insuficientes cuando el fenómeno se torna epidemiológico, y las instituciones no tienen la capacidad operativa para realizar la prevención, y contener los casos de parasuicidio e intento suicida. Es por esto que el estudio del suicidio a través de la autopsia psicológica, permite un acercamiento transdisciplinar desde la óptica de quien enfrenta el fenómeno a nivel familiar y social, aspecto relevante al momento de entender la relación entre causas y consecuencias de muchos suicidios. Dicho esto, los datos obtenidos de la aplicación de la AP, permiten crear estrategias, diseñar instrumentos, y aplicar programas donde es posible reducir en un alto grado el impacto social de su incidencia. Para el profesional social encargado de ejecutar la AP, la recolección de datos e información suficiente sobre el occiso y sus relaciones, favorece la conformación de un perfil del sujeto suicida, el cual ayuda a otros profesionales a diseñar planes preventivos e intervenciones significativas cada vez más seguras, al tiempo que configura una base analítica, desde la cual se puede hacer prevención temprana y atención de casos críticos, en las que el rol investigativo se torna propositivo, a través de la implementación de la conexión empática, humana y analítica, con quienes brindan la información de la persona que se quitó la vida.

A su vez los métodos y objetivos de éste elemento, posibilitan una aproximación conceptual y metodológica, sobre los factores psicosociales vinculados al acto suicida. Aspectos que en la rama de la Criminalística, permiten la evocación y exploración indirecta de la vida y la personalidad del sujeto fallecido. Lo anterior es dable a través de la valoración postmorten de la vida premorten, identificando las condiciones psicosociales de la victima, y la estructura y calidad de las relaciones psicosociales durante el tiempo de 
vida, además de su comportamiento y el estado mental. Dicho proceso implica la reconstrucción social, psicológica y biológica del suicida, para lo cual se requieren habilidades de entrevista, valoración psicológica a partir de la información recolectada, y el análisis clínico de la circunstancia psicobiológica de la victima. Operativamente la AP se vale de la técnica de investigación de escena de los hechos, a partir del análisis del cadáver en el lugar del suicidio, técnica de uso amplio en Criminalística (Núñez de Arco \& Huici, 2005). Igualmente existe una necesidad creciente de incluir información clínica, (psicológica, médica y psiquiátrica) en la $\mathrm{AP}$, que otorgue una mayor profundidad y exactitud al esclarecimiento de los casos, aspecto que debe complementarse con el análisis del porqué, el cómo y el para qué se produjo el suicidio, a fin de prevenir la consolidación de ideas suicidas y el posible parasuicidio en los familiares, lo que a su vez motiva la prevención de conductas de riesgo personal y social en personas vulnerables, pues los individuos que van a cometer el acto suicida, expresan verbal o simbólicamente sentimientos de inutilidad, soledad, desesperanza frente a la vida (Beck, Kovacs \& Weissman, 1979) con los que configuran ideas, planes, fantasías y aproximaciones a la muerte.

Consecuentemente los instrumentos para realizar la AP de acuerdo a Rubio (2011) son la historia clínica y la indagación de los procesos psicológicos del occiso, con un elevado rigor científico, espacio de análisis en el que se recolectan datos importantes de la vida y la muerte del suicida. De esta manera la entrevista a familiares, amigos y demás, se ejecuta de manera estricta, individual y en privado, evitando que las opiniones de cada individuo sean influenciadas por los otros, y así no aminorar la seguridad de la información recolectada. 
En éste sentido el MAPI genera una mayor confiabilidad en su praxis, por lo que se recomienda como herramienta de primera mano, para la evaluación de los casos de suicidio. Según la doctora Teresita García Pérez (1999) el MAPI arroja datos sociodemográficos, antecedentes patológicos, personales y familiares (toxicológicos), condiciones psicológicas del estado mental, datos acerca de la conducta individual y grupal, patrones de alimentación, sexualidad, sueño, actividad, lenguaje, conflictos (personales, familiares, económicos, antecedentes judiciales, duelos, etc.), como también rasgos de personalidad, cambio en los intereses y señales de suicidio, entre otros. Siendo estos aspectos muy útiles al momento de determinar el perfil de personalidad, las motivaciones personales y sociofamiliares y los posibles mensajes simbólicos, latentes en la ejecución de su propia muerte. 


\section{Referencias Bibliográficas}

Acevedo, NL, Pinzón, C. (1999). Propuesta para el Dictamen Forense en Casos de Muertes Dudosa Utilizando el procedimiento Denominado Autopsia Psicológica. Tesis de grado profesional no publicado, Pontificia universidad Javeriana, Santafé de Bogotá, 2-4. Recuperado el 16 de noviembre de 2012 de http://forumpotiguaranodesegurancapblica.blogspot.com/2010/02/psicologiaforense-v.html

Acosta, SA. (2011). La autopsia psicológica: técnica forense para el esclarecimiento del suicidio. Revista electrónica de psicología social, 2-4. Recuperado el 16 de Noviembre de 2012 de http://www.funlam.edu.co/revistas/index.php/poiesis/article/viewFile/88/61

Agamben, G. (1998). Homo sacer. El poder soberano y la nuda vida, trad. de A. Gimeno Cuspinera, Pre-Textos, Valencia.

Aja, EL. (2009). La autopsia psicológica en muertes por suicidio: algunos aspectos que los manuales no dicen. Revista Aquimindia, 4, 8-13. Recuperado el 20 de Agosto de 2012 de http://www.buscandoanimo.org/Descargas/Autopsia_psicol\%F3gica.pdf

Andrade, J. (2012). Aspectos psicosociales del comportamiento suicida en adolescentes. Revista electrónica de psicología, Iztacala, 15(2), 688-721. Recuperado el 20 de Agosto de 2012 de http://www.revistas.unam.mx/index.php/repi/article/view/32373

Annon, J. (1995). The PsycologicalAutposy.American Journal of Forensic Psychology, 13, 39-48. Recuperado el 10 de Septiembre de 2012 de http://psicologiajuridica.org/psj4.html

Appleby, L, Cooper, J, Amos T, Faragher, B. (1999). Psychological autopsy of suicides by people under 35. Br J Psychiatry, 175, 168-74.

Asgard, U. (1990). A psychiatric study of suicide among urban Swedish woman. Acta Psychiatrica Scandinavica, 82(2), 115-124.

Bárcena, E, Mardones, JM. (2006). La mirada excéntrica una educación desde la mirada de la víctima. La ética ante las víctimas. España: anthropos editorial.

Barradough, BM, Bunch, J, Nelson, B, Sainsbury, P. (1974). A hundred cases of suicide: clinical aspects. Br Journal Psychiatry, 125, 355-373. Recuperado el 10 de Septiembre de 2012 de http://annals.org/article.aspx?articleid=689202

Bateson, G. (1999). Pasos hacia una ecología de la mente. Editorial Lohlé-Lumen. 
Beck, AT, Kovacs, M, Weissman, A. (1979). Assessment of suicidal intention. The scale for Suicide Ideation. Journal Consult Clin Psychol, 47(2), 343-352. Recuperado el 10 de Septiembre de 2012 de http://www.ncbi.nlm.nih.gov/pubmed/469082

Burgos, A. (2006). La autopsia psicológica. Revista de Ciencias Jurídicas, 110, 69-87. Recuperado el 10 de octubre de 2012 de http://www.iij.ucr.ac.cr/archivos/publicaciones/revista/Revista\%20110.pdf

Cheng, ATA, Mann, AH, Chan, KA. (1997). Personality disorder and suicide. Br Journal Psychiatry, 170, 441-480.

Caballero, A, Recimil, MJ, Touriño, R, García, IL, Alonso, MC, Werlang, BS. (2010). Adaptación y validación de la Semi-Structured Interview for Psychological Autopsy (SSIPA) en Español. Actas Españolas de Psiquiatría, 38(6):332-339. Recuperado el 13 de enero de 2013 de http://www.actaspsiquiatria.es/repositorio/11/68/ENG/1168-ENG-332-339-591554.pdf

Código penal Colombiano. Por la cual se expide el Código de Procedimiento Penal. (Corregida de conformidad con el Decreto 2770 de 2004)". Recuperado el 10 de Noviembre de 2012 de http://www.alcaldiabogota.gov.co/sisjur/normas/Norma1.jsp?i=14787

Código de procedimiento penal Colombiano. Por la cual se expide el Código de Procedimiento Penal. (Ley 906 31/08/2004). Recuperado el 12 de enero de 2013 de http://www.elabedul.net/Documentos/ley_906_de_2004.pdf

Cross, T, Gust-Brey, K, Ball, P. (2002) A Psychological Autopsy of the Suicide of an Academically Gifted Student: Researchers' and Parents' Perspectives. Gifted Child Quarterly Vol. 46 (4), pp. 247 - 264. Recuperado el 10 de enero de 2013 de http://www.davidsongifted.org/db/Articles id 10337.aspx

Conwell, Y, Olsen, K, Caine, E \& Flannery, C. (1991). Suicide in later life: Psychological autopsy findings. International Psychogeriatrics, 3(1), 59-66.

Delgado, S, Esbec, E, Rodriguez, Gonzalez De Rivera \& Revuelta, JL. (1994). Psiquiatría Legal y Forense. Tomo I, cap. 50. España: Editorial Colex.

Durkheim, E. (1951). Suicide. Translated by Spaulding JA, Simpson G. New York, Free Press.

Ebert, B. (1991). Guide to Conducting a Psichological Autopsy. En K. Anchor. The Handbook of Medical Psychoterapy, 249-256. Recuperado el 10 de Septiembre de 2012 de csrp.hku.hk/files/855 3475 758.doc

Harris, EC, Barraclough, B. (1997). Suicide as an outcome for mental disorders. A metaanalysis. Br Journal Psychiatry, 170, 205-28. 
Herrero, JS, \& García, M. (2010) Suicidio. Revista de Psicoanálisis, Psicoterapia y Salud Mental. 3, 5-6 Recuperado el 10 de Septiembre de 2012 de http://psi.usal.es/rppsm/n72010/desantiago.pdf

Isometsa, ET. (2002). Estudios de Autopsia Psicológica: una revisión. Unidad de Investigación de los Trastornos del Estado de Ánimo y el Suicidio, 9, 11-18. Recuperado el 10 de Septiembre de 2012 de http://www.elmedicointeractivo.com/ap1/emiold/publicaciones/europeanpsy2002/1/ 11-18.pdf

Erdely, J. (2000). Suicidios colectivos rituales: un análisis interdisciplinario. Revista ciencia Ergo sum México, 7(1), 67-80. Recuperado el 10 de Septiembre de 2012 de http://redalyc.uaemex.mx/src/inicio/ArtPdfRed jsp?iCve=10401708

Forensis. (2011). Datos para la vida. Herramienta para la interpretación, intervención y prevención de lesiones de causa externa en Colombia. Instituto de medicina legal y ciencias forenses.

García, Pérez, T. (1993). La Autopsia Psicológica como método de estudio del suicida. Trabajo presentado en el congreso Internacional de ciencias forenses. La Habana: S.A.

García, Pérez, T. (1994). La autopsia psicológica como método de estudio del homicidio. Trabajo de Investigaciones del Instituto Superior de Ciencias Médicas de la Habana, 1994.

García, Pérez, T. (1995). La Autopsia Psicológica como método de estudio de las víctimas de homicidio. Encuentro Internacional de Técnicas Criminalísticas. La Habana: S.A.

García, Pérez, T. (1998). La autopsia psicológica en el suicidio Modalidad. Costa Rica: Heredia.

García, Pérez, T. (1999). La Autopsia Psicológica como Método de Estudio de Muertes Violentas. Instructivo para La Autopsia Psicológica (Utilizando el MAPI). La Habana: S.A.

García, Pérez T. (1999a). La autopsia psicológica en las muertes violentas. Rev Esp Med Leg 1999; XXIII (86-87), 75-82.

Gomez, V, Saenz, M. (2000). Aplicación de la autopsia psicológica en el proceso penal colombiano, 75-82. Recuperado el 10 de Septiembre de 2012 de http://www.javeriana.edu.co/biblos/tesis/derecho/dere1/Tesis35.pdf 
Heikkinen, M, Loenqvist, J. (1995). Recent life events in elderly suicide: A nationwide study in Finland. International Psychogeriatrics, 7(2), 287-300.

Isometsa, E, Henricksson, M, Heikkinen, M, Aro, H, Marttunen, M, Kuoppasalmi, K. (1996). Suicide Among Subjects WithPersonality Disorders. Am. J. Psychiatry. American psyquiatri plubishing: copyright.

Jiménez, I, Morales, M, Gelves, C. (2002). Análisis del suicidio a través de la autopsia psicológica. Revista Colombiana de Psiquiatría, 30(3), 271. Recuperado el 10 de Septiembre de 2012 de http://www.udea.edu.co/portal/page/portal/bibliotecaSedesDependencias/unidadesA cademicas/FacultadMedicina/BilbiotecaDiseno/Archivos/jornadasInvestigacion2/e$\underline{\text { h/eventos_vitales_adversos_y_suicidio_un_estudio_de_autops.pdf }}$

Jiménez, I. (2001). La autopsia psicológica como instrumento de investigación. Revista colombiana de psiquiatría, 30(3). Recuperado el 10 de Agosto de 2012 de http://www.buscandoanimo.org/autopsia-psicologica/

Juanatey, C. (1994). Derecho, suicidio y eutanasia. Madrid: Ministerio de Justicia e Interior. Centro de Publicaciones.

Kreitman, N. (1977). Parasuicide. London: Wiley Sons.

Litman, R. (1989). 500 Psychological Autopsies. Journal of Forensic Sciences, 34(3), 638640.

Morales, LA, Buela, GC, Bunce D, \& Jiménez, E. (2011) La autopsia Psicológica. Manual de Psicología Forense. Biblioteca Nueva: Madrid, 1, 18-22 Rec Recuperado el 12 de Noviembre de 2012 de uperado de http://es.calameo.com/read/000898836b1b95b3d8941

Mosquera, F. (2006). El comportamiento suicida, 80-81. Recuperado de http://www.psiquiatriabiologica.org.co/avances/vol7/doc/6 el comportamiento.pdf

Moya, J. (2007). La Conducta Suicida En Adolescentes sus Implicaciones en el ámbito de la justicia juvenil. Recuperado el 10 de Septiembre de 2012 de http://www.osamcat.cat/cat/Publicacions/Articles/ConductaSuicida.pdf

Núñez de Arco, J, Huici, T. (2005). El uso de la autopsia psicológica forense en el proceso penal. Identidad Jurídica - Revista del Ministerio Público de Bolivia, 1, 235-241. Recuperado el 10 de Diciembre de 2012 de http://www.nunezdearco.com/autopsiapsico.htm

Nuñez, LJ. (1999). Propuesta para el dictamen forense en casos de muerte dudosa utilizando el procedimiento denominado Autopsia Psicológica. Bogotá: Facultad de Psicología. Pontificia Universidad Javeriana. Tesis de grado. 
OMS. (2008). Suicidio en el mundo. "OMS: "En el mundo se produce un suicidio por depresión cada 40 segundos". Recuperado el 10 de Agosto de 2012 de http://www.who.int/mediacentre/news/releases/2008/pr37/es/index.html

OMS. (2010). Salud y desarrollo en el niño y el adolescente. ¿Por qué debemos invertir en la salud y el desarrollo de los adolescentes? Adolescencia. Recuperado el 15 de Octubre de 2012 de http://www.who.int/child adolescent health/topics/prevention care/adolescent/es/in dex.html

Peck, DL, Warner, K. (1995). Accident or suicide? Single vehicle car accidents and the intent hypothesis. Adolescence, 30(118), 463-72

Pérez, B. (1999). El suicidio, comportamiento y prevención. 15(2):196-217. Recuperado el 15 de Octubre de 2012 de http://bvs.sld.cu/revistas/mgi/vol15 2 99/mgi13299.htm

Pulido, R, Glez, R, Marco, G. Montes, O. (1990). La prevención primaria. Revista Psiquis, 90, 317-322. Recuperado el 15 de Octubre de 2012 de http://www.webs.ulpgc.es/revipsicoso/WEB/Numero_2/Pulido.pdf

Rubio, J. (2011). Psicología Forense. Recuperado el 15 de Octubre de 2012 de http://psicocormun.galeon.com/productos2484348.html

Sauceda, JM, Maldonado, JM. (2001). El suicidio en adolescentes ¿consecuencia de alguna forma de maltrato infantil? En Loredo Arturo. Maltrato en el niño. (pp.63-80). México: McGraw Hill

Sentencia C - 873 de 2003 M. P. Manuel José Cepeda Espinosa. Mediante auto del día diecinueve (19) de marzo de dos mil tres (2003). Congreso de la república-ejercicio de función constituyente/Nuevo sistema de investigación y juzgamiento en materia penal. Recuperado el 12 de enero de 2013 de http://www.corteconstitucional.gov.co/relatoria/2003/c-873-03.htm

Scott, CL, Swartz, E, Warburton, DO. (2006). Autopsia psicológica: resolver los misterios de la muerte. Psychiatr Clin N Am 29, 805-822. Clínicas Psiquiátricas de Norteamérica. Recuperado el 20 de diciembre de 2012 de http://www.elsevier.es/sites/default/files/elsevier/ficheros_catalogo/P/581/PRIM_P AGS_9788445817353.PDF

Shneidman, ES. (1967). The NIMH Center for Studies of Suicide Prevention. Bulletin Suicidology, 1, 2-7.

Shneidman, ES. (1981). The psychological autopsy. Suicide \& Life Threatening Behavior, 11, 325-340. Recuperado el 10 de Octubre de 2012 de http://onlinelibrary.wiley.com/doi/10.1111/j.1943-278X.1981.tb01009.x/abstract 
Shneidman, ES. (1994). Comment: The psychological autopsy. American Psychologist, 39, 75-76.

Strock, M. (2001). Depresión. National Institute of Health (NIH): Publicación, 04 -3561 SP.

Talcott, W. (2001). The Air Force Suicide Prevention Program. AFPAM, 21-23. Recuperado el 2 de Octubre de 2012 de http://209.212.22.88/data/rbr/19301939/1939/1939.10.19.pdf

Torres, M, Luna, J. (2008). Situación en Colombia. 3-10. Recuperado el 23 de Octubre de 2012 de http://www.alames.org/documentos/coltra.pdf

Torres, RI. (2007). Autopsia psicológica. Evaluación crítica y su aplicabilidad en el ámbito forense. Anuario de Psicología Jurídica, Chile. 17 (1), 111-130, recuperado el 10 de diciembre de la base de datos CSIC. BDDOC CSIC: Sumarios ISOC Psicología.

Tuesca, R, Navarro, E. (2003). Factores de riesgo asociados al suicidio e intento de suicidio. Revista Salud Uninorte, 17(1), 19-28

USAID \& Defensoría del pueblo. (2006). Técnicas de Investigación de la Defensa para el Sistema Penal Acusatorio Colombiano. Recuperado el 1 de diciembre de 2012 de http://200.82.81.155/escuela/recursos/Tecnicas-de-investigacion-de-la-defensa.pdf

Vidal, LE, Pérez, E, Borges, SA. (2006). Algunas consideraciones sobre la autopsia psicológica. Psicología.com. 10(1), 1-6.

Young, T. (1992). Procedures and Problems in Conducting a Psychological Autopsy. International Journal Offender Therapy and Comparative Criminology, 36, 43-52. Recuperado el 5 de Agosto de 2012 de http://psicologiajuridicaforense.wordpress.com/2011/01/05/autopsia-psicologica/ 
(a) (1)

Este texto está protegido por una licencia $\underline{\text { Creative Commons }} \underline{4.0}$.

Usted es libre para Compartir - copiar y redistribuir el material en cualquier medio o formato- y Adaptar el documento - remezclar, transformar y crear a partir del material — para cualquier propósito, incluso comercialmente, siempre que cumpla la condición de:

Atribución: Usted debe reconocer el crédito de una obra de manera adecuada, proporcionar un enlace a la licencia, e indicar si se han realizado cambios. Puede hacerlo en cualquier forma razonable, pero no de forma tal que sugiera que tiene el apoyo del licenciante o lo recibe por el uso que hace.

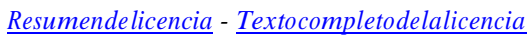

